\title{
Retinal hemorrhage as a unique ophthalmic manifestation of cerebral hyper perfusion syndrome
}

\author{
Eitan Heldenberg*, Arie Bass \\ Department of Vascular Surgery, Assaf Harofeh Medical Center, Sackler Faculty of Medicine, Tel Aviv University, Tel Aviv, Israel \\ Email: *eitan@heldenberg.name
}

Received 10 April 2013; revised 1 May 2013; accepted 9 May 2013

Copyright (c) 2013 Eitan Heldenberg, Arie Bass. This is an open access article distributed under the Creative Commons Attribution License, which permits unrestricted use, distribution, and reproduction in any medium, provided the original work is properly cited.

\begin{abstract}
Cerebral hyperperfusion syndrome (CHPS) is a rare, complication of carotid artery revascularization. Acute retinal hemorrhage is a very rare entity previously described as a manifestation of CHPS following carotid artery stenting (CAS), but to the best of our knowledge, not yet described as a complication of carotid surgery. An Ascending Aorta to bilateral Carotid bypass was performed in a 35-year-old woman with active Takayasu arteritis and $95 \%$ symptomatic stenosis of both common carotid arteries. Severe retinal hemorrhage appeared on the second post operative day combined with high blood pressure, brain edema on CT scan and grand mal seizures. It seems that fundoscopic examination following carotid revascularization of tight can be helpful in identifying those patients who develop symptoms suggesting of CHPS.
\end{abstract}

Keywords: Cerebral Hyper Perfusion Syndrome; Takayasu Arteritis; Retinal Hemorrhage

\section{INTRODUCTION}

Cerebral hyperperfusion syndrome (CHPS) first described by Sundt in 1981 is a potentially lethal complication, directly related to carotid revascularization [1]. CHPS presents with a wide spectrum of manifestations ranging from mild headaches to lethal intracerebral hemorrhage (ICH) [1,2]. The incidence of CHPS, as reviewed in various clinical series, has been $0.3 \%-2.7 \%$ [2].

We herein describe retinal hemorrhage as a manifestation of CHPS in a young patient with Takaysu's arteritis who underwent Ascending Aorta to bilateral distal Common Carotid Arteries bypass and developed brain edema as a manifestation of CHPS.

${ }^{*}$ Corresponding author.

\section{CASE PRESENTATION}

A 35-year-old previously healthy female, was admitted to the department of neurology with right hemiparesis and motor aphasia. Carotid duplex demonstrated diffuse thickening of both Common Carotid Arteries (CCA) without any pathology of the bifurcation or the Internal Carotid Arteries (ICA). Aortic arch and cervical vessel computerized tomographic angiography demonstrated 95\% stenosis of CCA, complete occlusion of the left Subclavian Artery (SCA) and $90 \%$ stenosis of the right SCA.

Based on the clinical presentation, imaging findings and elevated ESR (46), the patient was diagnosed as suffering from Takayasu Arteritis, and steroid therapy (Prednisone $20 \mathrm{mg}$ daily) was initiated. As all cervical arteries were either occluded or tightly stenosed, we preformed an Ascending Aorta to bilateral CCA bifurcation bypass using a $14 \times 7 \mathrm{~mm}$ bifurcated Dacron graft (Figure 1). Continuous Trans Cranial Doppler (TCD) exhibited a 1.5 and 2.5-fold (right and left, respectively) increase in middle cerebral artery (MCA) peak systolic velocities immediately after carotid clamp removal. This MCA flow velocity augmentation persisted for one hour following the revascularization. In the recovery room the patient had elevated blood pressure, 194/96, followed by grand mal seizures, which resolved with Diazepam and Epanutin treatment. On post operative day 3 the patient complained of having blurred vision accompanied by high blood pressure, 184/94, diffuse headache and grand mal seizures. MRI demonstrated diffuse brain edema (Figure 2). Retinal hemorrhage was diagnosed on fundoscopic examination (Figure 3). The patient was treated by Aspirin, Clopidogrel and Epanutin (to prevent the seizures recurrence). The patient recovered completely with no neurological residue.

\section{DISCUSSION}

CHPS is a well known entity among vascular surgeons 


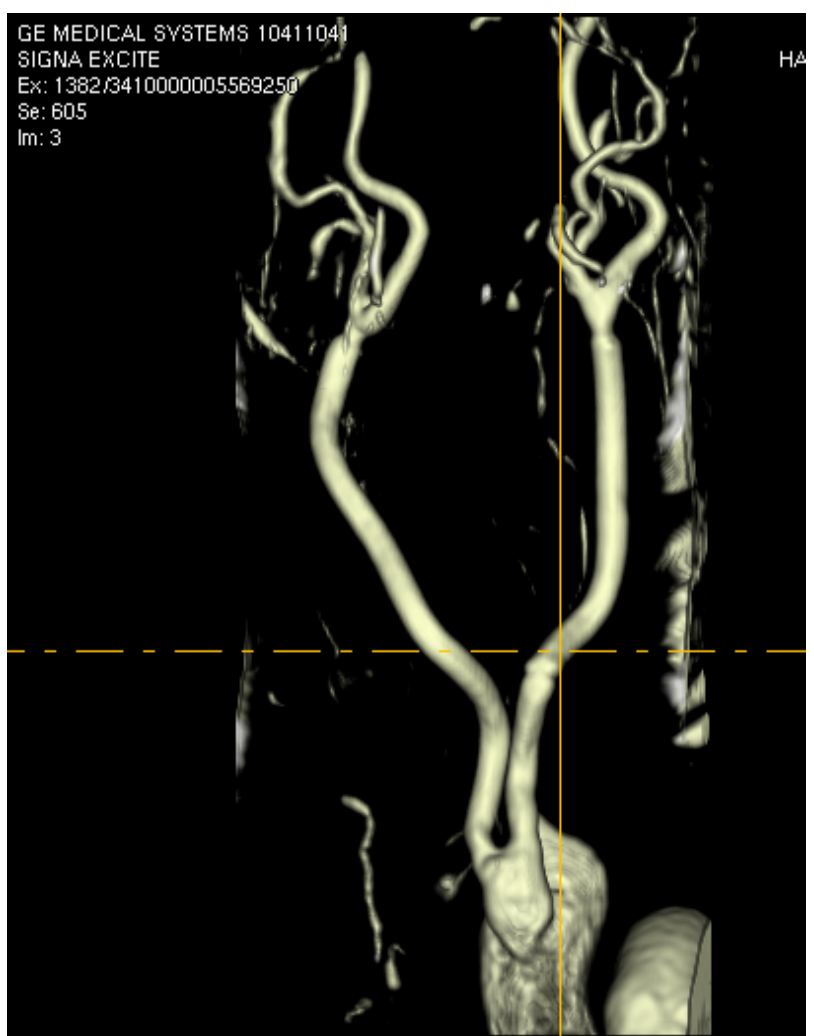

Figure 1. MRI examination visualizing the Aorto-Bi-Carotid (ABC) bypass. The graft is emerging off the ascending aorta and its limbs were anastomosed end-to-end to the distal common carotid arteries.

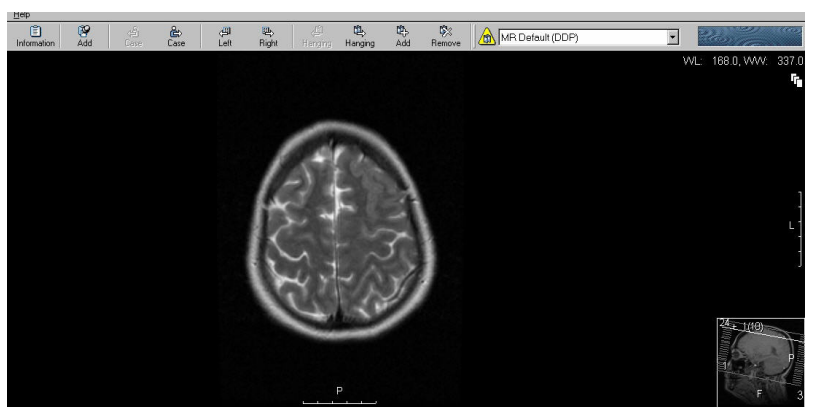

(a)

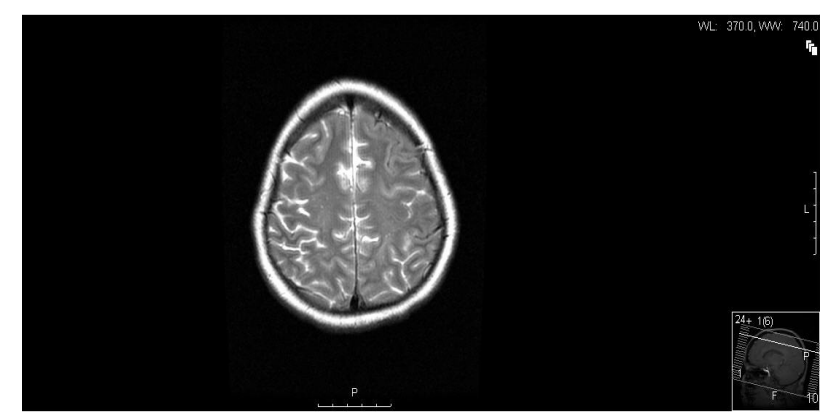

(b)

Figure 2. Brain MRI four days after cerebral revascularization is demonstrating vasogenic edema (VE) in the left frontal lobe (a). Five weeks later the edema has disappeared (b).

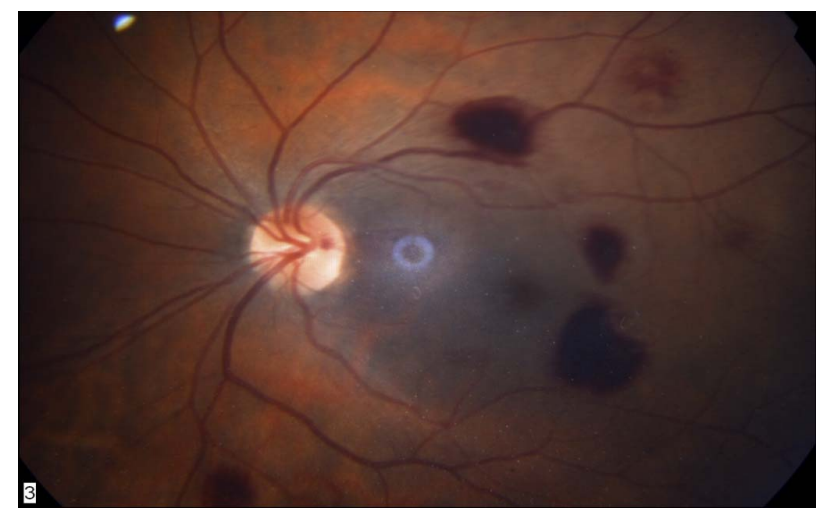

(a)

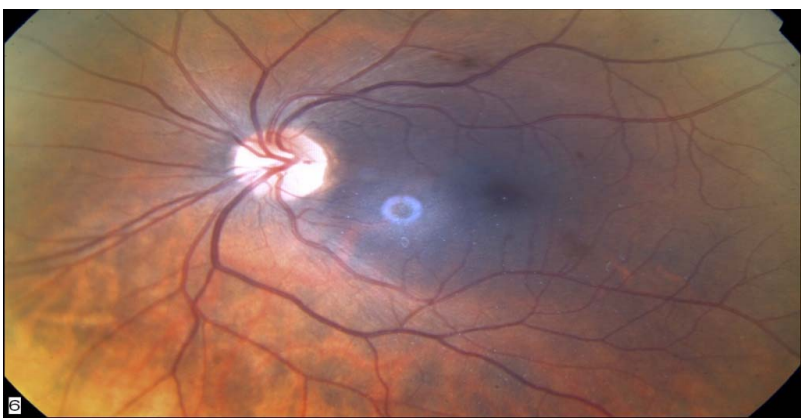

(b)

Figure 3. Fundoscopic examination four days after revascularization. Multiple retinal hemorrhages are demonstrated in both eyes (a). Three weeks later most hemorrhages have been absorbed (b).

who treat carotid artery disease, either by endarterectomy or stenting, with an average incidence of $0.3 \%-2.7 \%$ [1].

It seems that the risk of developing CHPS is increased among patients with severe, diffuse cerebrovascular disease, such that the worse the occlusive disease and the more cerebral vessels involved, the higher the probability of developing CHPS.

The pathogenesis, although obscure, is attributed to the failure of autoregulation of cerebral blood flow in chronically hypoperfused brain hemispheres following revascularization. Maximal dilatation of cerebral arterioles secondary to marginal arterial perfusion may cause loss of cerebral vascular auto regulation. This, in conjunction with uncontrolled hypertension, may result in brain edema or intracerebral hemorrhage (ICH) once normal blood flow has been restored [1,3]. The intracerebral edema may further worsen the hypertension resulting in further hyperperfusion.

Although the literature is replete with descriptions of CHPS, there is a lack of literature regarding its ophthalmic manifestation CHPS [4]. Only one recent publication regarding retinal hemorrhage following carotid artery stenting (CAS) as a manifestation of CHPS has been published [5]. 
The symptoms of impaired visual acuity in our patient could have been attributed to vasogenic brain edema, which was demonstrated by MRI. Previous case reports described an association between reversible cortical blindness and vasogenic cerebral edema, among patients with severe postpartum preeclampsia [6].

Our patient presented with fundoscopic evidence of binocular retinal hemorrhages, coinciding with brain vasogenic edema. This linkage may reflect a rather unrecognized entity of bleeding within neuronal tissue, related to capillary leak in the setting of uncontrolled hypertension. Furthermore, routine use of Aspirin and Clopidogrel and utilization of anticoagulants during surgery may further increase the risk of bleeding.

This unusual case illustrates that fundoscopic examination may be useful in diagnosing CHPS after carotid revascularization.

\section{REFERENCES}

[1] Sundt, T.M., Sharbourgh, F.W., Piepgras, D.G., Kearns, T.P., Messick, J.M. and O’Fallon, W.M. (1981) Correlation of cerebral blood flow and electroencephalographic changes during Endarterectomy: With results of surgery and hemodynamic of cerebral ischemia. Mayo Clinic
Proceedings, 56, 533-543.

[2] Ogasawara, K., Mikami, C., Inoue, T. and Ogawa, A. (2004) Delayed cerebral hyperperfusion syndrome caused by prolonged impairment of cerebrovascular auto regulation after carotid endarterectomy: Case report. Neurosurgery, 54, 1258-1262. doi:10.1227/01.NEU.0000120064.55339.F9

[3] Krapanayiotides, T., Meuli, R., Devuyst, G., PiechowskiJozwiak, B., Dewarrat, A., Ruchat, P., Segger, V.L. and Bogousslasky, J. (2005) Post carotid endarterectomy hyperperfusion or reperfusion syndrome. Stroke, 6, 21-36.

[4] Nicholas, G.G., Hashemi, H., Gee, W. and Reed, F.J. (1993) The cerebral hyperperfusion syndrome: Diagnostic value of ocular pneumoplethysmography. Journal of Vascular Surgery, 17, 690-695. doi:10.1016/0741-5214(93)90112-Y

[5] Kim, D.Y., Kim, B.M., Park, H. and Chung, J. (2011) Retinal hemorrhage as an initial sign of cerebral hyperperfusion syndrome after carotid artery stenting. Acta Neurochirurgica, 154, 75-77. doi:10.1007/s00701-011-1194-z

[6] Do, D.V., Rismondo, V. and Nyguyen, Q.D. (2002) Reversible cortical blindness in preeclampsia. American Journal of Ophthalmology, 134, 916-918. doi:10.1016/S0002-9394(02)01753-1 